\title{
Lesions of Nucleus Accumbens Disrupt Learning about Aversive Outcomes
}

\author{
Geoffrey Schoenbaum and Barry Setlow \\ Department of Psychological and Brain Sciences, Johns Hopkins University, Baltimore, Maryland 21218
}

\begin{abstract}
Nucleus accumbens (NAcc) is critical for encoding and using information regarding the learned significance of cues predictive of reward. However, its role in processing information about cues predictive of aversive outcomes is less well studied. Here, we examined the effects of NAcc lesions in an odor-guided discrimination task in which rats use odor cues predictive of either appetitive or aversive outcomes to guide responding. Rats with sham or neurotoxic lesions of NAcc were trained on a series of two-odor discrimination problems. Performance on each problem was assessed by monitoring accuracy of choice behavior and by measuring latency to respond for fluid reinforcement after odor sampling. After acquisition of four problems, rats were trained on serial reversals of the final problem. Rats with NAcc lesions exhibited normal choice performance relative to controls on both acquisition and reversal of the discrimination problems (indeed, lesioned rats exhibited a mild facilitation on the first discrimination problem). Despite normal choice performance, however, lesioned rats failed to show normal changes in response latency during discrimination learning, particularly on trials involving the aversive outcome. These findings are consistent with a deficit in processing cue- outcome associations. These results are compared with those obtained from studies of basolateral amygdala and orbitofrontal cortex lesions in this task and suggest that NAcc integrates the motivational value of both appetitive and aversive cues to bias or modulate the vigor of subsequent responding.
\end{abstract}

Key words: accumbens; olfactory; discrimination; reversal; striatum; orbitofrontal; amgydala

\section{Introduction}

The nucleus accumbens (NAcc) has been implicated in integrating motivational information to guide behavior (Mogenson et al., 1980; Kelley, 1999; Parkinson et al., 2000a). Damage to NAcc results in deficits in a variety of tasks in which the acquired motivational properties of predictive cues are used to guide or bias subsequent responding (Parkinson et al., 2000b; Hall et al., 2001; Setlow et al., 2002), and neurons in NAcc fire to cues predictive of both appetitive and aversive outcomes (Williams et al., 1993; Kawagoe et al., 1998; Tremblay et al., 1998; Ravel et al., 1999; Hassani et al., 2001; Carelli, 2002; Cromwell and Schultz, 2003; Setlow et al., 2003). These findings support a role for NAcc in using information about acquired cue value to modulate the vigor of subsequent responding (Parkinson et al., 2000a; Cardinal et al., 2002; de Borchgrave et al., 2002).

Anatomically, NAcc is well situated to play this role, because it is a site of convergence for numerous sources of motivational information. The basolateral complex of the amygdala (ABL) and orbitofrontal cortex (OFC), which send strong, direct projections to NAcc (Groenewegen et al., 1990; McDonald, 1991; Haber et al., 1995), have been implicated in a range of emotional and motivational processing, including acquisition and expression of

Received May 30, 2003; revised Sept. 9, 2003; accepted Sept. 9, 2003.

This work was supported by National Institute on Aging Grant K08-AG00882 and National Institute on Drug Abuse Grant R01-DA015718 to G.S. and by National Institute of Mental Health Grant F32-MH12699 to B.S. We thank Dr. Gorica Petrovich for help with photography and Dr. Michela Gallagher for her support (R01-MH60179).

Correspondence should be addressed to Dr. Geoffrey Schoenbaum, University of Maryland School of Medicine, Department of Anatomy and Neurobiology, 685 West Baltimore Street, Health Science Facility-1 Room 280K, Baltimore, MD 21201. E-mail: schoenbg@schoenbaumlab.org.

Copyright $\odot 2003$ Society for Neuroscience $\quad$ 0270-6474/03/239833-09\$15.00/0 motivational properties of predictive cues (Davis, 1992; Rolls, 1996; Everitt et al., 2000; Gallagher, 2000; Schoenbaum and Setlow, 2001), and neurons in ABL and OFC fire to stimuli predictive of appetitive and aversive outcomes (Thorpe et al., 1983; Nishijo et al., 1988; Muramoto et al., 1993; Quirk et al., 1995; Schoenbaum and Eichenbaum, 1995; Rolls et al., 1996; Schoenbaum et al., 1999; Tremblay and Schultz, 1999; Maren, 2000). These neurophysiological findings, combined with recent reports regarding NAcc (Williams et al., 1993; Blazquez et al., 2002; Horvitz, 2002; Setlow et al., 2003; Yanagimoto and Maeda, 2003), suggest that NAcc encodes both appetitive and aversive information; yet historically, NAcc has been implicated primarily in learning about reward. Indeed, behavioral work comparing OFC, $\mathrm{ABL}$, and NAcc is available mainly from appetitive tasks (Everitt et al., 1991; Hatfield et al., 1996; Cardinal et al., 2001; Mobini et al., 2002; Setlow et al., 2002).

Here, we evaluated the effects of bilateral lesions of NAcc on acquisition and reversals in a go, no-go odor discrimination task in which we previously demonstrated appetitive and aversive encoding properties of NAcc neurons (Setlow et al., 2003). In this task, thirsty rats learn to discriminate between two odors to obtain an appetitive sucrose solution and to avoid an aversive quinine solution. A key feature of this task is that appropriate behavior requires learning about the aversive outcome. We assessed performance on the task by examining both choice behavior (measured as the accuracy of go and no-go responses) and latency to respond at the fluid well after odor sampling. These two measures may reflect different associative processes (Holland and Straub, 1979; Sage and Knowlton, 2000), and previous experiments have shown that $\mathrm{ABL}$ and $\mathrm{OFC}$ lesions differentially affect 
different performance measures in this task (Schoenbaum et al., 2003). The goals of the present experiment were to examine the effects of NAcc damage on learning in this setting, and, in particular, to examine the role of NAcc in learning about cues predictive of aversive outcomes.

\section{Materials and Methods}

Subjects. All procedures were conducted at Johns Hopkins University in accordance with University and National Institutes of Health guidelines. The subjects consisted of 23 male Long-Evans rats (300-350 gm) obtained from Charles River Laboratories (Wilmington, MA). Rats were housed individually in the Ames Hall vivarium at Johns Hopkins University on a $12 \mathrm{hr}$ light/dark cycle (lights on at 8:00 A.M.) with ad libitum access to food and water except during testing. During testing, rats were given ad libitum access to water for $\sim 30 \mathrm{~min} / \mathrm{d}$ after testing. All testing was performed during the light phase of the cycle.

Before odor discrimination training, all rats in the current study received training in Pavlovian conditioning experiments involving visual and auditory conditioned stimuli paired with food reward (Hatfield et al., 1996; Petrovich et al., 2002). This training occurred in a separate apparatus in a location different from that used in the present experiment using different cues and reinforcers than the current paradigm. Approximately 2 weeks elapsed between the completion of these experiments and the start of odor discrimination training. During this time, rats remained in their home cages and received periodic handling and ad libitum food and water.

Surgery. Surgery took place before the Pavlovian conditioning experiments described above ( $\sim 80 \mathrm{~d}$ before the start of odor discrimination training). Rats were anesthetized with isoflurane and placed in a stereotaxic frame (David Kopf Instruments, Tujunga, CA) fitted with an isoflurane gas anesthesia system. A midline incision was made, the skin and periosteum were retracted, and holes were drilled through the skull over the lesion sites. A 30 gauge needle attached by a length of plastic tubing to a $10 \mu \mathrm{l}$ microsyringe (Hamilton, Reno, NV) mounted on a syringe pump (Sage Instruments, Boston, MA) was used for intracerebral infusions. Lesions were made using $15 \mu \mathrm{g} / \mu \mathrm{l}$ quinolinic acid (Sigma, St Louis, MO) in phosphate buffer vehicle, buffered with Tris base. Phosphate buffer vehicle alone was used to make sham control lesions. For intracerebral infusions, the needle was lowered to the flat skull coordinates from bregma (anteroposterior, $+2.1 \mathrm{~mm}$; mediolateral, $\pm 1.6 \mathrm{~mm}$; and dorsoventral, $-7.2 \mathrm{~mm}$ from the skull surface), and $0.4 \mu \mathrm{l}$ of quinolinic acid or vehicle was infused over $3 \mathrm{~min}$. After the infusion, the needle was left in place for $4 \mathrm{~min}$ to allow for diffusion. Fourteen rats received quinolinic acid infusions, and nine rats received vehicle infusions. After surgery, the incision was closed with wound clips and antibiotic ointment was applied to the wound site. Rats were monitored during recovery from anesthesia and on subsequent days for behavioral disturbances and signs of infection.

Apparatus. Behavioral testing was conducted using a set of four identical chambers. Each chamber was constructed of aluminum and measured $\sim 45 \mathrm{~cm}$ per side but with sloping walls narrowing to an area $30 \times$ $30 \mathrm{~cm}$ at the floor. An exhaust fan was located on the upper back wall, and the front wall was hinged to open outward and provide access to the interior. Two panel lights were located on the right wall of the chamber. The test chambers were located in a small room $(3 \times 3 \mathrm{~m})$, the door to which remained closed during behavioral sessions. Two speakers located in the corners of the room broadcast output from a white noise generator to mask extraneous noise.

Each chamber was connected to a Pentium II $266 \mathrm{MHz}$ computer for behavioral control and data acquisition. Sessions were conducted using a program written in $\mathrm{C}++$ and running in DOS. Registers located on a DT2817 input-output board (Data Translation, Marlboro, MA) were used to control and detect events. Events (animal- and computerinitiated) were time stamped using values from a CIO-DIO-CTR3 clock timer board (Computer Boards, Middleboro, MA), capable of microsecond resolution. These data were saved for later analysis.

Odors were selected from a set of compounds obtained from International Flavors and Fragrances (New York, NY), which were classified
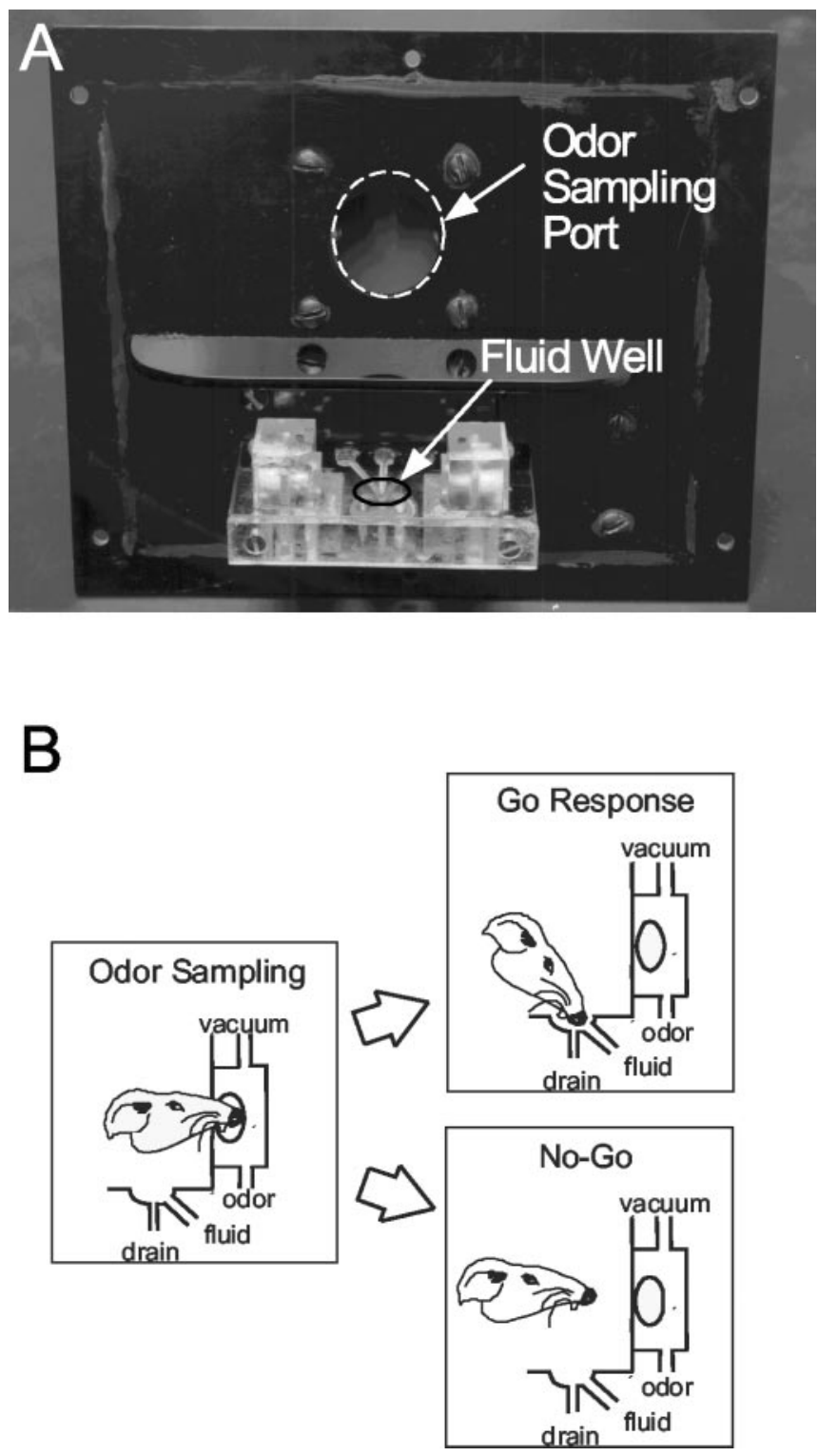

Figure 1. Illustration of training apparatus and behaviors in the task. $A$, Photograph of the polycarbonate panel removed from the operant chamber to show the odor sampling port (white dashed circle) and the fluid delivery well (black circle). $B$, Schematic drawings illustrating the sequence of behaviors in the go, no-go olfactory discrimination task using the apparatus in $A$.

subjectively into categories. Discrimination problems consisted of odors from different categories (fruity, spicy, herbal, etc.), and categories did not repeat in sequentially presented discriminations. These odor compounds were diluted 1:20 in propylene glycol. The set of diluted odors used in the discrimination problems in this experiment were isolated on a removable cartridge connected to a system of solenoids and flowmeters to allow each odor to be delivered individually to the training chamber. All tubing and valves associated with an odor were dedicated to that odor to prevent any cross-contamination between cues, and each training chamber had an identical set of odor cues (Schoenbaum, 2001).

Odors were presented at an odor delivery port located in a polycarbonate panel (Fig. 1A), bolted into an opening in the right wall of each operant box below the panel lights. The odor sampling port consisted of a $2.5-\mathrm{cm}$-diameter opening. A photobeam across the opening registered nosepokes into the port. Behind this port was a small hemicylinder where odorized air streams could be presented when a rat nosepoked at the odor port. Odors were delivered through tubing connected to the base of the hemicylinder behind the sampling port.

Before each trial, an odor was selected for delivery by opening a sole- 
noid valve that allowed a clean air stream to pass over one of the odor solutions on the removable cartridge. The odorized air stream at 1.5 $1 /$ min was brought to a vacuum dump behind the odor port. This vacuum drew at $2.0 \mathrm{l} / \mathrm{min}$ and was also attached by a $1 \mathrm{~cm}$ line to the hemicylinder behind the odor port, resulting in a negative flow of $0.5 \mathrm{l} / \mathrm{min}$ out of the hemicylinder at all times except during odor delivery. Odor delivery was initiated by closing the vacuum after detection of a nosepoke at the odor port, thereby causing the odorized air stream to be diverted into the hemicylinder behind the port with an onset latency of $\sim 25 \mathrm{msec}$. Odor delivery was terminated by opening the vacuum when the rat left the odor port. During odor presentation, a second vacuum line drawing at $2.0 \mathrm{l} / \mathrm{min}$ from the top of the hemicylinder prevented the odor from entering the training chamber.

Fluids were delivered to a depression located in a ledge just below the odor sampling port (Fig. $1 \mathrm{~A}$ ). Responses at the fluid well were detected by a photobeam passing parallel to the ledge $\sim 1 \mathrm{~mm}$ above the well depression. The well depression was tapped with a single line at the bottom. This central line opened into four lines concealed deeper in the ledge. Two of these lines were used to deliver sucrose and quinine solutions. A third line was used to deliver water to clean the well between trials, and the fourth line was used as a vacuum-assisted drain line. Solenoid valves controlled the delivery of each fluid and the operation of the drain.

Behavioral testing. Before the start of odor discrimination training, rats were shaped to nosepoke at the odor port to receive a water reward in the fluid well. The rats were gradually shaped to hold their snout in the odor port for a period of $250 \mathrm{msec}$ before odor delivery and $500 \mathrm{msec}$ after odor delivery and to make a response to the fluid well within $3000 \mathrm{msec}$ to receive a reward. Once shaped to this procedure, the first phase of odor discrimination training began.

During odor discrimination training, the rats were presented with a series of odor problems. Each odor discrimination problem consisted of two odors $(\mathrm{S} 1, \mathrm{~S} 2)$. One odor signaled a positive outcome $(\mathrm{S} 1+)$, indicating that a response at the fluid well would result in delivery of a $5 \%$ sucrose solution. The other odor signaled a negative outcome (S2-), indicating that the same response would result in delivery of a $0.02 \mathrm{M}$ quinine solution. Trials were signaled to the rat by illumination of the panel lights inside the box. When these lights were on, a nosepoke into the odor port (Fig. $1 B$ ) resulted in delivery of the preselected odor cue. The rat terminated odor sampling by leaving the odor port. The rat then had $3 \mathrm{sec}$ to make a go response at the fluid well (Fig. $1 B$ ). If a response was detected, then fluid was delivered to the well. After a response, fluid remained in the well and the panel lights remained on until the rat left the fluid well, then the fluid was removed by activating the drain line, and the lights were extinguished to end the trial. If the rat did not respond at the fluid well within $3 \mathrm{sec}$ of exiting the odor port, the trial was counted as a no-go (Fig. $1 B$ ) and the panel lights were extinguished. Typically, rats began each new discrimination problem by responding to both odors, and then they learned to withhold responding on negative trials to avoid the aversive quinine (Fig. $1 \mathrm{~B}$ ). Intertrial intervals were $4 \mathrm{sec}$ after correct responses and $9 \mathrm{sec}$ after incorrect responses. During the intertrial intervals, the fluid well was flushed with water twice, and an odor was selected for presentation during the next trial.

Odor discrimination training was divided into two phases. In the first phase, rats were required to learn a series of four two-odor discrimination problems (D1-D4). Note that the first odor problem (D1) served as a "shaping" problem in which the rats were introduced to odors and to the sucrose and quinine reinforcers for the first time in the context of the task. Training continued on this and each of the subsequent nonshaping odor problems until the rat met a criterion of 18 correct responses in a moving block of 20 trials. Rats were run for $\sim 1 \mathrm{hr}$ each day or until this criterion was achieved. When an odor problem was acquired, training began on the next problem in the series in the session the next day.

Once the first four odor problems (D1-D4) were acquired, the rats began the second phase of training. In the second training phase, the rats were required to learn a series of reversals, in which the contingencies signaled by the odor cues in a single discrimination problem were altered. This phase began with presentation of the most recently acquired odor problem (D4) using the same contingencies that were used in initial training $(\mathrm{S} 1+/ \mathrm{S} 2-)$. Rats were considered to have reached accurate retention performance on this discrimination by meeting a behavioral criterion of 18 correct responses in a moving block of 20 trials. At this point, the response contingencies were reversed, provided that the rat had maintained $80 \%$ performance over a block of 60 trials preceding reversal. This secondary performance requirement ensured that all rats were equally proficient on the odor problem before reversal, but it was not considered in calculating the number of trials to reach behavioral criterion performance. Training on the reversed problem $(\mathrm{S} 1-/ \mathrm{S} 2+)$ continued until the behavioral criterion was met again.

After this first reversal was completed, the contingencies for the same odor cues were reversed a second time in the same manner. The reversed discrimination problem $(\mathrm{S} 1-/ \mathrm{S} 2+)$ was presented, and the rats were required to demonstrate retention of this problem with these contingencies by achieving the behavioral criterion of 18 correct responses in a moving block of 20 trials. When these criteria were met, the problem was reversed immediately back to the original contingencies $(\mathrm{S} 1+/ \mathrm{S} 2-)$, provided that the rat had maintained $80 \%$ performance over a block of 60 trials preceding reversal. Training on this re-reversal continued until each rat met the behavioral criterion again.

During behavioral testing, the rats' relative preferences for the two outcomes (sucrose and quinine) were assessed. For this test, the rats were maintained on fluid restriction just as during training, but instead of a daily training session, the rats were presented with bottles containing sucrose and quinine in their home cages. Both outcomes were presented at the same concentrations used in testing, and the rats were allowed access to the two bottles simultaneously for $30 \mathrm{~min}$. Consumption of each outcome was measured.

Histology. After completion of all behavioral testing, rats were given an overdose of pentobarbital $(100 \mathrm{mg} / \mathrm{kg})$ and perfused intracardially with $0.9 \%$ saline followed by $4 \%$ formaldehyde. Brains were removed and stored in $4 \%$ formaldehyde for $24 \mathrm{hr}$, followed by $30 \%$ sucrose in $4 \%$ formaldehyde until slicing. The brains were sliced on a freezing microtome, and $40 \mu \mathrm{m}$ coronal sections were collected through the area of NAcc. These sections were mounted on glass slides, stained with thionin, and coverslipped with Permount. Lesion placements were verified under a light microscope and drawn onto plates adapted from the atlas of Paxinos and Watson (1997).

Data analysis. Acquisition on each discrimination problem in the first phase of the study was evaluated by calculating the trials required to reach the behavioral criterion for each animal (18 of 20 correct). These data were analyzed by ANOVA with repeated measures (lesion $\times$ odor problem). A response latency measure obtained during the acquisition of the three nonshaping discrimination problems (D2-D4) was also analyzed. Latency to respond at the fluid well after odor sampling was calculated for positive and negative go trials. For the analysis of this behavioral measure, trials that occurred before criterion were divided into an early and a late phase of acquisition, the border between which was defined by the occurrence of the sixth error in the session. The difference in latency on positive and negative trials was analyzed by ANOVA with repeated measures (lesion $\times$ odor problem $\times$ training phase). Performance in the reversal phase, which consisted of two serial reversals, was evaluated by comparing the trials required to meet the behavioral criterion (18 of 20 correct trials) when retaining and reversing the D4 odor problem with either the original or the altered contingencies. These data were analyzed by ANOVA with repeated measures (lesion $\times$ contingency $\times$ reversal), for which contingency referred to whether the rat was performing on the $\mathrm{D} 4$ discrimination problem with the original $(\mathrm{S} 1+/ \mathrm{S} 2-)$ or the novel contingencies $(\mathrm{S} 1-/ \mathrm{S} 2+)$ and reversal referred to whether it was reacquiring the same contingencies as in the previous session or acquiring a reversal of those contingencies. Fluid consumption in a preference test was compared for the sucrose and quinine outcomes by ANOVA with repeated measures (lesion $\times$ outcome). Statistics were computed using the ANOVA-multivariate ANOVA module in Statistica (Statsoft, Tulsa $\mathrm{OK})$, and individual comparisons were made using contrasts $(p<0.05)$. 

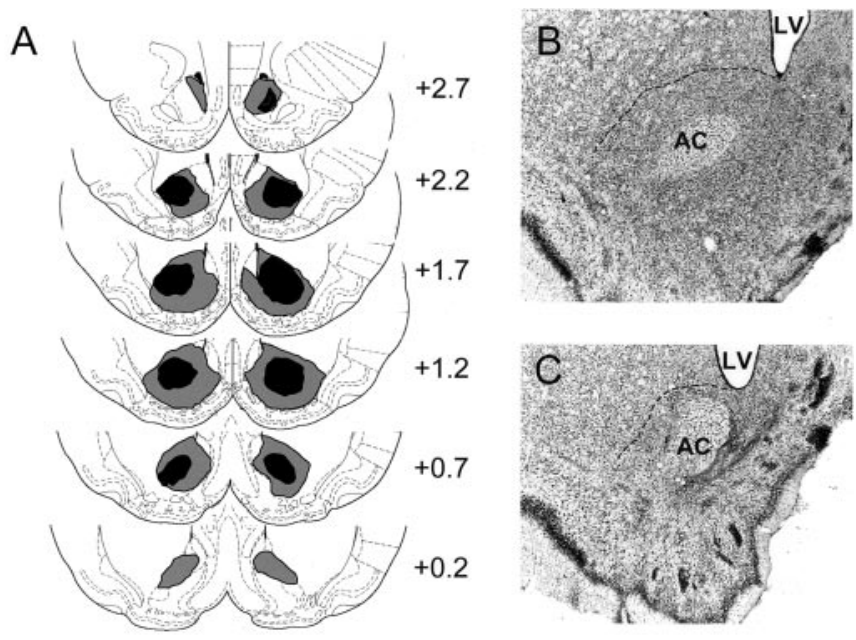

Figure 2. Drawings and photomicrographs showing the NAcc in sham and lesioned subjects. $A$, Drawings depict the largest (gray areas) and smallest (black areas) lesions from animals used in the experiment. $B$, Photomicrograph of a coronal section taken through NAcc in a sham control rat. C, Photomicrograph of a coronal section through NAcc in a lesioned rat. Note the considerable degree of tissue shrinkage in the lesioned section relative to the control section. AC, Anterior limb of the anterior commissure; LV, lateral ventricle. Dashed lines indicate approximate borders of NAcc. Plates are adapted from the atlas of Paxinos and Watson (1997).

\section{Results \\ Histology}

Of the 14 rats that underwent surgery to create bilateral NAcc lesions, one was excluded because of bilateral damage to piriform cortex anterior to NAcc (note that this rat also failed to acquire any of the odor discrimination problems). In the remaining 13 rats, the lesions generally encompassed the entire anteroposterior extent of NAcc, including the core subregions in all cases. In 11 of these 13 rats, damage also encompassed much of the shell subregion, including the lateral, ventral, and medial shell, although the dorsomedial tip of the shell was spared in all cases. The remaining two rats had lesions restricted to the core subregion; behavior in these two rats did not differ from the other lesioned rats on any of the measures detailed in this report. Damage outside the boundaries of NAcc consisted of occasional damage to the caudateputamen overlying the core subregion and minor damage to posterior ventral pallidum. Other than minor mechanical damage along the track of the injection needle, there was no observable damage in sham-lesioned control rats.

The largest and smallest lesions included in the analysis are illustrated in Figure 2A (the smallest lesion illustrated is one of the two in which the lesion was restricted to the core subregion). A representative section from a sham control and lesioned rat is shown in Figure 2, $B$ and $C$, respectively. Note that there was often a considerable degree of tissue shrinkage in the lesioned rats, resulting in rearrangement of structures surrounding NAcc (compare the position of the anterior limb of the anterior commissure relative to the lateral ventricle and medial surface of the brain in Fig. 2B,C).

\section{Sucrose and quinine preferences}

The relative preferences of control and NAcc-lesioned rats between the sucrose and quinine solutions used in the discrimination task are shown in Figure 3. Both groups of rats showed a strong preference for sucrose over quinine, and there was no difference between groups. A two-factor ANOVA (treatment $\times$ sucrose-quinine outcome) revealed a significant main effect of

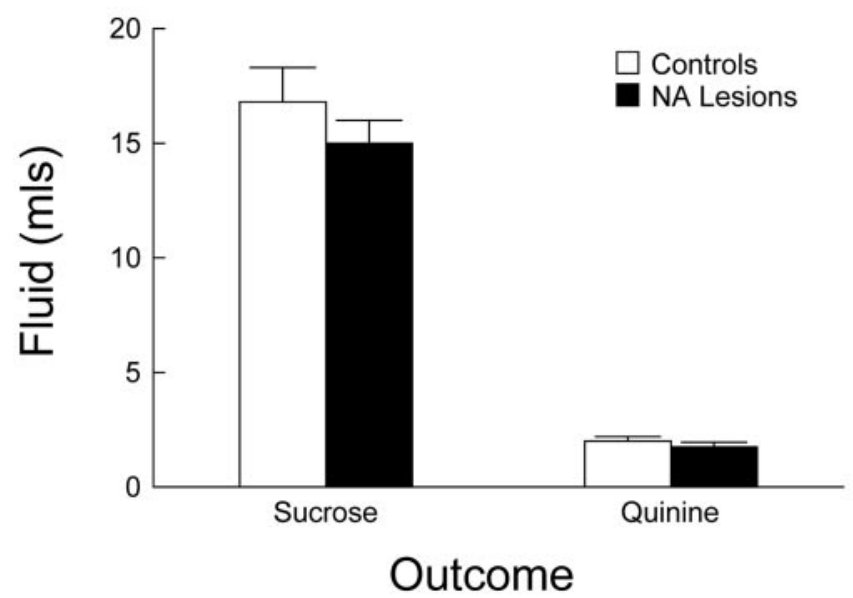

Figure 3. Relative preferences for $5 \%$ sucrose and $0.02 \mathrm{~m}$ quinine by NAcc (NA)-lesioned (black bars) and control (white bars) rats. Both groups of rats showed a strong preference for the sucrose over the quinine solution, and there was no effect of treatment on this relative preference. Error bars indicate SEM.

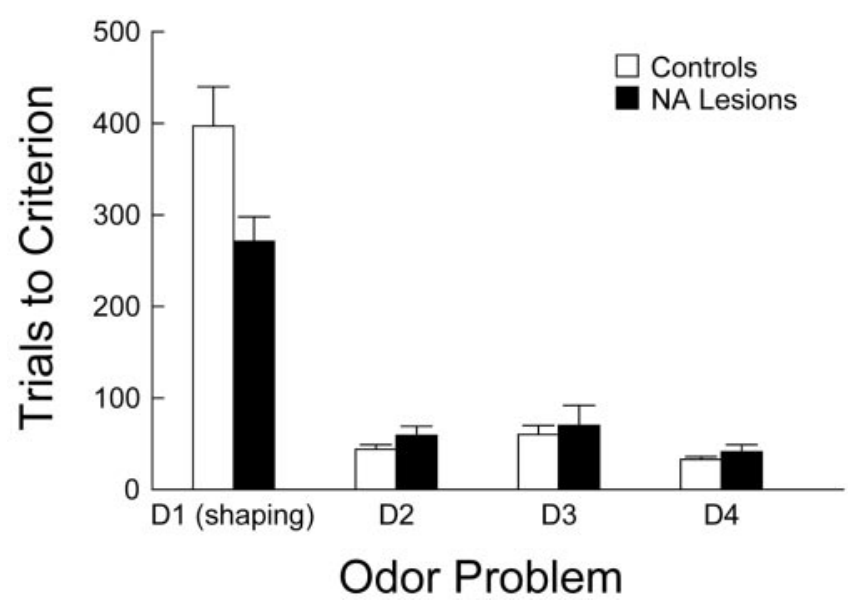

Figure 4. Acquisition of successive odor discrimination problems by NAcc (NA)-lesioned (black bars) and control (white bars) rats. The rate of acquisition of each odor discrimination problem (D1-D4) is represented as the trials it took for each rat to meet a criterion of 18 correct responses in a moving block of 20 trials. NAcc-lesioned rats showed a significant facilitation on acquisition of the first shaping problem. There was no effect of lesion on acquisition of any of the subsequent odor discriminations or on the improvement observed across successive problems. Error bars indicate SEM.

sucrose-quinine outcome $\left(F_{(1,19)}=284 ; p<0.001\right)$ and no effect or interaction involving treatment group. One NAcc-lesioned rat failed to exhibit a preference for sucrose over quinine, consuming $2 \mathrm{ml}$ of quinine compared with only $3 \mathrm{ml}$ of sucrose during the 30 min test period. As a result of this abnormal $(>2$ SD from the mean sucrose consumption of the lesioned group) sucrose preference, this animal was excluded from additional testing.

Acquisition of new discriminations in rats with NAcc lesions The acquisition of each odor discrimination problem by the rats in the NAcc-lesioned and control groups is shown in Figure 4. All rats, regardless of lesion condition, achieved criterion performance on each of the four discrimination problems. A two-factor ANOVA (lesion $\times$ odor problem) revealed no effect of lesion condition on acquisition, although there was a significant main effect of odor problem $\left(F_{(3,57)}=102 ; p<0.01\right)$, such that acquisition on the first shaping problem (D1) required more trials than 
on subsequent nonshaping problems (D2-D4). In addition, there was a significant interaction between lesion and odor prob$\operatorname{lem}\left(F_{(3,57)}=6.10 ; p<0.01\right)$. Contrast testing revealed that this effect was attributable to a significant facilitation of the lesioned rats' acquisition of the initial shaping problem $\left(F_{(3,57)}=6.83 ; p<\right.$ $0.05)$. Interestingly, this facilitation was even more pronounced in the two rats with lesions restricted to the core region of NAcc (average trials-to-criterion, 164), although their performance was not statistically different from the rate of acquisition in the other NAcc-lesioned rats $(p=0.12)$. When the first problem was excluded, there were no significant effects or interactions.

Subsequent analyses focused on performance during acquisition of these three odor problems (D2-D4). The performance of lesioned and control rats was similar across these problems. This similarity in the performance of the two groups was evident when the precriterion trials were divided into early and late blocks as described in Materials and Methods for the purpose of response latency analysis. The numbers of trials in each block did not differ significantly between groups, and the groups performed at a similar level of accuracy across the different trial blocks. NAcc-lesioned rats performed at 59\% correct and $72 \%$ correct in the early and late blocks, respectively, and at $93 \%$ in the postcriterion block, whereas controls performed at $61 \%$ correct in the early block, $74 \%$ correct in the late block, and $91 \%$ in the postcriterion block.

\section{Response latency during acquisition of new discriminations}

Previous observations in our laboratory (Schoenbaum et al., 2000) indicate that rats normally develop a difference in latency to enter the fluid well after sampling odors that signal positive versus negative outcomes. Responses are made more rapidly after sampling a "positive" (sucrose-paired) odor, whereas responses are made more slowly after sampling a "negative" (quininepaired) odor over the course of precriterion training. This behavioral phenomenon is evident in the results presented for the control group in Figure 5, in which a latency difference emerged during precriterion trials. In contrast, rats with NAcc lesions failed to develop this difference in response latency. A threefactor ANOVA (lesion $\times$ odor problem $\times$ phase) revealed a significant main effect of lesion $\left(F_{(1,19)}=4.63 ; p<0.05\right)$ and a significant interaction between lesion and phase $\left(F_{(2,38)}=8.36\right.$; $p<0.001)$. There was no impact of odor problem on the lesion effect, so this factor was collapsed in evaluating the interaction between lesion and latency difference. Subsequent contrast testing revealed that the difference in response latency (negative minus positive) for control rats increased significantly from -29 msec during the early phase to $85 \mathrm{msec}$ during the late phase and to $220 \mathrm{msec}$ during the postcriterion phase. In contrast, NAcclesioned rats exhibited a nonsignificant change from 46 to 26 msec from the early to the late phase of precriterion training and $56 \mathrm{msec}$ postcriterion.

A separate consideration of responding on positive and negative trials (Fig. 5, inset) revealed that although intact and lesioned rats both increased their speed of responding on positive trials across the training phases, the lesioned rats failed to decrease their speed of responding on negative trials. Consistent with this interpretation, a three-factor ANOVA (lesion $\times$ odor problem $\times$ phase) comparing latency on the positive trials revealed a significant main effect of phase $\left(F_{(2,38)}=30.4 ; p<0.001\right)$ but no significant effects or interactions involving lesion (note that the interaction between lesion and phase did approach significance at $p<0.09$, suggesting that controls tended to become faster in their responding on positive trials). A similar ANOVA comparing latency on negative trials revealed a significant interaction between

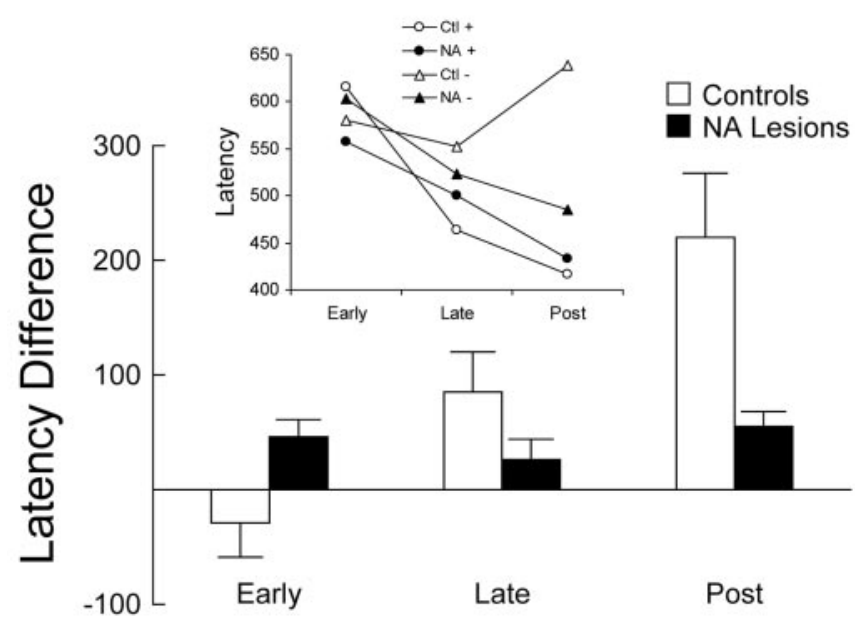

Training Phase

Figure 5. Latency (in milliseconds) to respond at the fluid well after odor sampling for NAcc (NA)-lesioned (black bars) and control (white bars) rats across the three phases of the odor discrimination problems. The main figure shows the difference in response latency, which was calculated as the average response latency on negative minus positive trials within each phase, averaged for the second (D2), third (D3), and fourth (D4) discrimination problems. Actual response latency on positive and negative trials is shown in the inset. No-go trials, in which rats made no response for $3000 \mathrm{msec}$, were excluded from the analysis. NAcc-lesioned rats failed to develop the learning-related latency difference exhibited by control ( $\mathrm{Ctl})$ rats. The failure to develop this latency difference primarily reflected abnormal changes in response latency on negative trials. Error bars indicate SEM.

lesion and phase $\left(F_{(2,38)}=4.41 ; p<0.05\right)$. These findings are consistent with motivational modulation of responding in intact rats on both positive and negative trials, which is absent in the discriminative responding of NAcc-lesioned rats. This effect is particularly pronounced on aversive trials, because the effect of the motivational information is to antagonize the normal "warm-up" effect, which increases the speed of responding as the rats start a session. The trend observed in positive trials suggests that this is the case. Thus, although intact rats exhibited very different patterns of responding on positive versus negative trials, NAcc-lesioned rats facilitated responding during training regardless of the likely outcome of the trial.

\section{Performance during serial reversals}

The performance of control and NAcc-lesioned rats across two serial reversals is shown in Figure 6. Reversal training used the final odor discrimination problem (D4) from the first training phase. Rats were required to demonstrate retention of the original contingencies $(\mathrm{S} 1+/ \mathrm{S} 2-)$ by meeting the behavioral criterion of 18 of 20 correct and then acquire a reversal of those contingencies $(\mathrm{S} 1-/ \mathrm{S} 2+)$ by meeting the same criterion. Subsequently, the rats were required to demonstrate retention of the altered contingencies $(\mathrm{S} 1-/ \mathrm{S} 2+)$ and then acquire a reversal back to the original contingencies $(\mathrm{S} 1+/ \mathrm{S} 2-)$. As described in Materials and Methods, rats were required to be at $80 \%$ performance before reversal (there were no differences between sham and lesioned rats in the amount of training necessary to meet this requirement).

As shown in Figure 6, rats with NAcc lesions performed similarly to controls on these serial reversals, regardless of the contingency (original vs novel) that was presented in the reversal. In agreement with this description, a three-factor ANOVA (lesion $\times$ contingency $\times$ reversal) showed significant main effects 


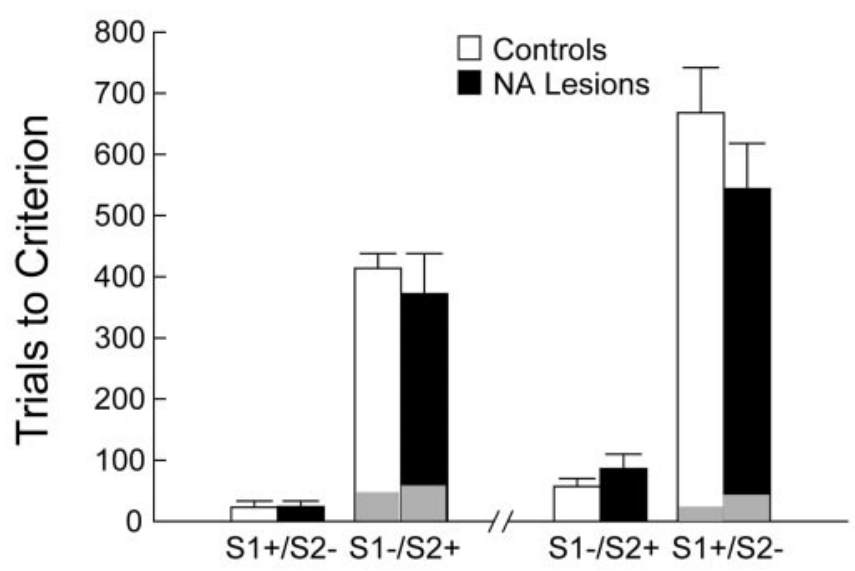

Contingencies

Figure 6. Choice performance across serial reversals of the final odor discrimination problem (D4) by NAcc (NA)-lesioned (black bars) and control (white bars) rats. Performance is shown for both the retention and reversal phases of training, represented as the trials required for each rat to meet a criterion of 18 correct responses in a moving block of 20 trials. Gray areas on reversal days indicate the trials required to reach $50 \%$ performance as a measure of perseveration on the old contingencies. NAcc-lesioned rats were not impaired at acquiring reversals, regardless of whether the reversed odor discrimination problem was composed of new contingencies ( $\mathrm{S} 1$ - I $\mathrm{S} 2+$ ) or was a return to the original contingencies $(\mathrm{S} 1+/ \mathrm{S} 2-)$, nor was there any effect of lesion on the tendency to perseverate on reversal days. Error bars indicate SEM.

of reversal $\left(F_{(1,19)}=120 ; p<0.001\right)$ and contingency $\left(F_{(1,19)}=\right.$ $16.4 ; p<0.001)$, but no main effect or any interaction involving lesion $(F$ values $<1.8$; $p$ values $>0.19)$. Thus, rats with NAcc lesions were unimpaired in acquiring and retaining serial reversals of an odor problem regardless of whether the reversal involved the original or altered contingencies.

There was also no effect of NAcc lesions on the degree of perseveration on the previous contingencies after reversal. Examination of performance after reversal revealed that NAcc-lesioned and control rats both reached chance performance of 50\% (10 of 20 correct) after reversal at similar rates (Fig. 6, gray areas). A two-factor ANOVA (lesion $\times$ reversal session) comparing the trials required to reach $50 \%$ performance after reversal ( 10 of 20 trials correct in a moving block of 20 trials) showed no significant main effect or interaction with lesion $(F$ values $<1.3$; $p$ values $>$ $0.27)$. There was a significant main effect of reversal session $\left(F_{(1,19)}=7.50 ; p<0.05\right)$, indicating that both lesioned and control rats required fewer trials to reach $50 \%$ performance on the second reversal than on the first one.

\section{Discussion}

This report contains two main findings regarding the effects of NAcc lesions on performance in a go, no-go odor discrimination task. First, lesions abolished normal changes in response latencies during learning. Whereas intact rats developed faster responses on positive trials and slower responses on negative trials as they learned discrimination problems, rats with NAcc lesions failed to show these differential changes in latency. This failure was particularly manifest on negative trials. Second, this impairment occurred in the absence of impairments in choice behavior, either before or after reversal, despite the requirement that rats inhibit strong prepotent tendencies to respond at the fluid well.

\section{NAcc is important for learning about aversive outcomes}

The most notable finding is that NAcc is important for modulating responding to reflect the motivational value of aversive out- comes. Although most studies of NAcc have used cues predictive of rewarding events, emerging evidence implicates NAcc in processing of aversive information (Williams et al., 1993; Salamone et al., 1997; Parkinson et al., 1999; Becerra et al., 2001; Blazquez et al., 2002; Horvitz, 2002; Reynolds and Berridge, 2002; Setlow et al., 2003; Yanagimoto and Maeda, 2003). This evidence indicates that NAcc may be generally involved in the use of cues predictive of biologically significant outcomes, either rewarding or aversive.

Our findings are consistent with this proposal. Rats with NAcc lesions failed to modulate responding to the odor cues to reflect the associated outcomes. This deficit was primarily but not entirely attributable to a failure to make slower responses on the negative trials, demonstrating that the effects of NAcc lesions were not restricted to learning about rewarding outcomes. A general role for NAcc in processing affective information is consistent with the encoding properties of neurons in NAcc in this task, which fire to both the positive and negative cues and outcomes and exhibit encoding characteristics that are essentially identical for oppositely valenced information (Setlow et al., 2003).

\section{Role of NAcc in modulating the vigor of responding}

As noted previously (Mogenson et al., 1980), the NAcc is positioned between limbic structures critical to affective processing and motor circuits that guide responding. This positioning makes it ideally situated to integrate a variety of motivational information to modulate behavior. The effect of NAcc lesions in the odor discrimination task is consistent with a modulatory role; NAcc was critical for modulating the vigor of responding but did not seem to be critical to determining which response was selected. This result is in agreement with several studies indicating that the strength of responding is affected by processing in NAcc (Hauber et al., 2000; Parkinson et al., 2000b; Cardinal et al., 2002) (but see Brown and Bowman, 1995). Effects on the speed or vigor of responding may reflect information about the incentive value of the associated outcome (Holland and Straub, 1979; Sage and Knowlton, 2000) and, thus, the impairment observed here may be attributable to deficits in processing some aspect of the odoroutcome associations in the task, either in the associative process itself or in the use of such information to influence behavior. This suggestion is consistent with findings that damage to ABL and OFC, which project to NAcc and are critical to cue-outcome associative processes in other contexts (Hatfield et al., 1996; Gallagher et al., 1999; Baxter et al., 2000), produces latency deficits in the odor discrimination task similar to those observed here (Schoenbaum et al., 2003).

Notably, in this same paradigm, we demonstrated a subpopulation of "rapidly selective" neurons in NAcc that developed differential firing to the odor cues at the same time that the rats exhibited these response latency differences (Setlow et al., 2003). These neurons also appeared to encode outcome-related information later in each trial, suggesting that the selective firing during cue sampling might encode information about the associated outcomes. The current findings suggest that these representations of outcome value in NAcc are critical for modulating the strength or speed of responding in the task.

It is equally important that NAcc was not critical for selecting the appropriate response, as evidenced by the preserved discriminative performance. A previous examination of the effects of NAcc lesions on odor discrimination learning found no effect on choice performance during acquisition but did report an impairment on reversals (Ferry et al., 2000). The discrepancy between this study and our results may reflect the size of the lesions in the study by Ferry et al. (2000), which extended into ventral palli- 
dum. Ventral pallidal lesions had effects similar to NAcc lesions in that study, so the effects of NAcc lesions on reversals may have reflected collateral damage. In addition, their rats received substantial presurgical training, which may encourage the use of NAcc-dependent mechanisms. Similar effects have been observed previously in comparisons of studies using OFC lesions (Eichenbaum et al., 1983; Dias et al., 1997; Schoenbaum et al., 2002).

There are several important implications of this preserved choice performance. First, preserved performance suggests that the effects of lesions on response latency were not attributable to general learning, sensory, or motivational deficits. Second, preserved performance indicates that appropriate responses can be selected even in the absence of NAcc function. Discriminative responding can be mediated by associative mechanisms different from those that mediate changes in response latency (Sage and Knowlton, 2000), suggesting that NAcc is critically involved in the latter but not the former processes. Third, preserved performance in the context of a go, no-go task indicates that NAcc lesions do not cause a general inability to inhibit responses, because successful performance both before and after reversal requires the inhibition of strongly learned prepotent tendencies acquired over many trials.

The preserved performance is interesting in light of our previous report on neural encoding in NAcc in this task (Setlow et al., 2003). In addition to the aforementioned population of rapidly selective neurons, a separate "slowly selective" subpopulation of neurons was identified in NAcc that only became selective during accurate discriminative performance, as if guiding the responses in the task. The present results indicate that if these neurons are important for response selection, their contribution is not critical to the ability of the rat to select the appropriate response; other brain systems must be able to compensate for the loss of this subpopulation in NAcc in our task. In this context, it is intriguing that studies have found a variety of effects of NAcc damage on discrimination learning and reversal in different tasks (Robbins et al., 1990; Reading et al., 1991; Burk and Mair, 2001), suggesting that particular task requirements may determine whether these representations of response selection in NAcc are critical for performance.

The absence of a general deficit in inhibiting responding is of particular interest in light of evidence that lesions of the core subregion of NAcc lead to "impulsive choice" of a small immediate reward over a large delayed reward (Cardinal et al., 2001). Unlike the current study, in which choice performance was based primarily on the ability to avoid responding for an aversive outcome, that report compared rats' abilities to choose between different amounts of a single appetitive outcome. In addition, the paradigm required the rats to discount the reward value to account for a delay before reward delivery. These differences highlight the fact that impulsivity and response inhibition are not unitary phenomena; rather, they reflect the operation of a variety of component processes (Evenden, 1999). The present results suggest that impulsive behavior is not a general consequence of NAcc damage but may be limited to settings that place a particular emphasis on one of these component processes. For example, this deficit may be particularly pronounced when the task requires the ability to select between multiple choices on the basis of relatively subtle differences in outcome value, a requirement emphasized by the task of Cardinal et al. (2001) but not by that in the present report. Indeed, acquiring and even reversing a go, no-go discrimination, as assessed by choice behavior, may be particularly insensitive to deficits in this ability.

\section{Implications for OFC-ABL interactions and the role of NAcc}

This report characterizes the effects of NAcc lesions in a task used previously to examine the effects of $\mathrm{OFC}$ and $\mathrm{ABL}$ lesions (Schoenbaum et al., 2003). A comparison of these studies reveals shared and unique contributions of each region to task performance. Bilateral lesions of each area abolished changes in response latency differences observed in intact rats. This effect is consistent with the known involvement of these areas in learning about the motivational significance of cues (Mogenson et al., 1980; Davis, 1992; Rolls, 1996; Kelley, 1999; Everitt et al., 2000; Gallagher, 2000; Parkinson et al., 2000a; Schoenbaum and Setlow, 2001; Cardinal et al., 2002) and with evidence suggesting that latency to respond to cues is particularly sensitive to learning about the incentive value of associated outcomes (Holland and Straub, 1979; Sage and Knowlton, 2000). Recording experiments are also consistent with the involvement of all three regions in modulating speed of responding during learning in this task. In both $\mathrm{ABL}$ and NAcc neurons, we observed changes in firing to cues in this task coincident with changes in response latency (Schoenbaum et al., 1999; Setlow et al., 2003), and OFC neurons fire in expectation of the outcomes at the same time that changes in response latency are observed (Schoenbaum et al., 1998). These findings are consistent with suggestions that this system is critical for the encoding and use of stimulus-outcome associations to modulate behavior.

In contrast, these regions appear to be less important for discriminative responding. None of the lesions affected rats' abilities to acquire the initial series of discrimination problems, indicating that rats with damage to this system are still able to discriminate between cues and respond to them appropriately. Importantly, this task requires rats to inhibit prepotent go responses, so damage within this system does not appear to result in a generic difficulty with response inhibition, a function often ascribed to OFC and, more recently, to NAcc.

Finally, each region has a unique effect on reversal learning. OFC lesions impair reversal learning, ABL lesions cause a mild deficit in learning and retaining a reversal of the original contingencies, and NAcc lesions have no apparent effect. These results suggest that although rapid reversal learning depends critically on functions within OFC, perhaps augmented by interactions with $\mathrm{ABL}$, this capability does not require outflow from OFC-ABL to NAcc. This finding is consistent with the idea that NAcc modulates but does not necessarily determine or select responses to cues. The capacity of networks within OFC to mediate flexible behavior (perhaps interacting with $\mathrm{ABL}$ to integrate outcomerelated information) must be able to be implemented through connections with regions other than NAcc.

\section{References}

Baxter MG, Parker A, Lindner CC, Izquierdo AD, Murray EA (2000) Control of response selection by reinforcer value requires interaction of amygdala and orbitofrontal cortex. J Neurosci 20:4311-4319.

Becerra L, Breiter HC, Wise RA, Gonzalez RG, Borsook D (2001) Reward circuitry activation by noxious thermal stimuli. Neuron 32:927-946.

Blazquez PM, Fujii N, Kojima J, Graybiel AM (2002) A network representation of response probability in the striatum. Neuron 33:973-982.

Brown VJ, Bowman EM (1995) Discriminative cues indicating reward magnitude continue to determine reaction time of rats following lesions of the nucleus accumbens. Eur J Neurosci 7:2479-2485.

Burk JA, Mair RG (2001) Effects of dorsal and ventral striatal lesions on delayed matching trained with retractable levers. Behav Brain Res 122:67-78.

Cardinal RN, Pennicott DR, Sugathapala CL, Robbins TW, Everitt BJ (2001) 
Impulsive choice induced in rats by lesions of the nucleus accumbens core. Science 292:2499-2501.

Cardinal RN, Parkinson JA, Hall J, Everitt BJ (2002) Emotion and motivation: the role of the amygdala, ventral striatum, and prefrontal cortex. Neurosci Biobehav Rev 26:321-352.

Carelli RM (2002) Nucleus accumbens cell firing during goal-directed behaviors for cocaine vs "natural" reinforcement. Physiol Behav 76:379-387.

Cromwell HC, Schultz W (2003) Effects of expectations for different reward magnitudes on neuronal activity in primate striatum. J Neurophysiol 89:2823-2838.

Davis M (1992) The role of the amygdala in conditioned fear. In: The amygdala: neurological aspects of emotion, memory, and mental dysfunction (Aggleton JP, ed), pp 255-306. Chichester, UK: Wiley.

de Borchgrave R, Rawlins JN, Dickinson A, Balleine BW (2002) Effects of cytotoxic nucleus accumbens lesions on instrumental conditioning in rats. Exp Brain Res 144:50-68.

Dias R, Robbins TW, Roberts AC (1997) Dissociable forms of inhibitory control within prefrontal cortex with an analog of the Wisconsin Card Sort Test: restriction to novel situations and independence from "online" processing. J Neurosci 17:9285-9297.

Eichenbaum H, Clegg RA, Feeley A (1983) Reexamination of functional subdivisions of the rodent prefrontal cortex. Exp Neurol 79:434-451.

Evenden JL (1999) Varieties of impulsivity. Psychopharmacology (Berl) 146:348-361.

Everitt BJ, Morris KA, O’Brien A, Robbins TW (1991) The basolateral amygdala-ventral striatal system and conditioned place preference: further evidence of limbic-striatal interactions underlying reward-related processes. Neuroscience 42:1-18.

Everitt BJ, Cardinal RN, Hall J, Parkinson JA, Robbins TW (2000) Differential involvement of amygdala subsystems in appetitive conditioning and drug addiction. In: The amygdala: a functional analysis (Aggleton JP, ed), pp 353-390. New York: Oxford UP.

Ferry AT, Lu XC, Price JL (2000) Effects of excitotoxic lesions in the ventral striatopallidal-thalamocortical pathway on odor reversal learning: inability to extinguish an incorrect response. Exp Brain Res 131:320-335.

Gallagher M (2000) The amygdala and associative learning. In: The amygdala: a functional analysis (Aggleton JP, ed), pp 311-330. New York: Oxford UP.

Gallagher M, McMahan RW, Schoenbaum G (1999) Orbitofrontal cortex and representation of incentive value in associative learning. J Neurosci 19:6610-6614.

Groenewegen HJ, Berendse HW, Wolters JG, Lohman AH (1990) The anatomical relationship of the prefrontal cortex with the striatopallidal system, the thalamus and the amygdala: evidence for a parallel organization. Prog Brain Res 85:95-118.

Haber SN, Kunishio K, Mizobuchi M, Lynd-Balta E (1995) The orbital and medial prefrontal circuit through the primate basal ganglia. J Neurosci 15:4851-4867.

Hall J, Parkinson JA, Connor TM, Dickinson A, Everitt BJ (2001) Involvement of the central nucleus of the amygdala and nucleus accumbens core in mediating Pavlovian influences on instrumental behavior. Eur J Neurosci 13:1984-1992.

Hassani OK, Cromwell HC, Schultz W (2001) Influence of expectation of different rewards on behavior-related neuronal activity in the striatum. J Neurophysiol 85:2477-2489.

Hatfield T, Han JS, Conley M, Gallagher M, Holland P (1996) Neurotoxic lesions of basolateral, but not central, amygdala interfere with Pavlovian second-order conditioning and reinforcer devaluation effects. J Neurosci 16:5256-5265.

Hauber W, Bohn I, Giertler C (2000) NMDA, but not dopamine D2, receptors in rat nucleus accumbens are involved in guidance of instrumental behavior by stimuli predicting reward magnitude. J Neurosci 20:6282-6288.

Holland PC, Straub JJ (1979) Differential effects of two ways of devaluing the unconditioned stimulus after Pavlovian appetitive conditioning. J Exp Psychol Anim Behav Process 5:65-78.

Horvitz JC (2002) Dopamine gating of glutaminergic sensorimotor and incentive motivational input signals to the striatum. Behav Brain Res 137:65-74.

Kawagoe R, Takikawa Y, Hikosaka O (1998) Expectation of reward modulates cognitive signals in the basal ganglia. Nat Neurosci 1:411-416.
Kelley AE (1999) Neural integrative activities of nucleus accumbens subregions in relation to learning and memory. Psychobiology 27:198-213.

Maren S (2000) Auditory fear conditioning increases CS-elicited spike firing in lateral amygdala neurons even after extensive overtraining. Eur J Neurosci 12:4047-4054.

McDonald AJ (1991) Organization of the amygdaloid projections to the prefrontal cortex and associated striatum in the rat. Neuroscience 44:1-14.

Mobini S, Body S, Ho MY, Bradshaw CM, Szabadi E, Deakin JF, Anderson IM (2002) Effects of lesions of the orbitofrontal cortex on sensitivity to delayed and probabilistic reinforcement. Psychopharmacology (Berl) 160:290-298.

Mogenson GJ, Jones DL, Yim CY (1980) From motivation to action: functional interface between the limbic system and the motor system. Prog Neurobiol 14:69-97.

Muramoto K, Ono T, Nishijo H, Fukuda M (1993) Rat amygdaloid neuron responses during auditory discrimination. Neuroscience 52:621-636.

Nishijo H, Ono T, Nishino H (1988) Single neuron responses in alert monkey during complex sensory stimulation with affective significance. J Neurosci 8:3570-3583.

Parkinson JA, Robbins TW, Everitt BJ (1999) Selective excitotoxic lesions of the nucleus accumbens core and shell differentially effect aversive Pavlovian conditioning to discrete and contextual cues. Psychobiology 27:256-266.

Parkinson JA, Cardinal RN, Everitt BJ (2000a) Limbic cortical-ventral striatal systems underlying appetitive conditioning. Prog Brain Res 126:263-285.

Parkinson JA, Willoughby PJ, Robbins TW, Everitt BJ (2000b) Disconnection of the anterior cingulate cortex and nucleus accumbens core impairs Pavlovian approach behavior: further evidence for limbic cortical-ventral striatopallidal systems. Behav Neurosci 114:42-63.

Paxinos G, Watson C (1997) The rat brain in stereotaxic coordinates. New York: Academic.

Petrovich GD, Setlow B, Holland PC, Gallagher M (2002) Amygdalohypothalamic circuit allows learned cues to override satiety and promote eating. J Neurosci 22:8748-8753.

Quirk GJ, Repa JC, LeDoux JE (1995) Fear conditioning enhances shortlatency auditory responses of lateral amygdala neurons: parallel recordings in the freely behaving rat. Neuron 15:1029-1039.

Ravel S, Legallet E, Apicella P (1999) Tonically active neurons in the monkey striatum do not preferentially respond to appetitive stimuli. Exp Brain Res 128:531-534.

Reading PJ, Dunnett SB, Robbins TW (1991) Dissociable roles of the ventral, medial and lateral striatum on the acquisition and performance of a complex visual stimulus-response habit. Behav Brain Res 45:147-161.

Reynolds SM, Berridge KC (2002) Positive and negative motivation in nucleus accumbens shell: bivalent rostrocaudal gradients for GABA-elicited eating, taste "liking"/“disliking" reactions, place preference/avoidance, and fear. J Neurosci 22:7308-7320.

Robbins TW, Giardini V, Jones GH, Reading P, Sahakian BJ (1990) Effects of dopamine depletion from the caudate-putamen and nucleus accumbens septi on the acquisition and performance of a conditional discrimination task. Behav Brain Res 38:243-261.

Rolls ET (1996) The orbitofrontal cortex. Philos Trans R Soc Lond B Biol Sci 351:1433-1443.

Rolls ET, Critchley HD, Mason R, Wakeman EA (1996) Orbitofrontal cortex neurons: role in olfactory and visual association learning. J Neurophysiol 75:1970-1981.

Sage JR, Knowlton BJ (2000) Effects of US devaluation on win-stay and win-shift radial maze performance in rats. Behav Neurosci 114:295-306.

Salamone JD, Cousins MS, Snyder BJ (1997) Behavioral functions of nucleus accumbens dopamine: empirical and conceptual problems with the anhedonia hypothesis. Neurosci Biobehav Rev 21:341-359.

Schoenbaum G (2001) Olfactory learning and the neurophysiological study of rat prefrontal function. In: CRC series: methods in chemosensory research (Nicolelis MAL, Simon SA, eds), pp 371-427. Boca Raton, FL: CRC.

Schoenbaum G, Eichenbaum H (1995) Information coding in the rodent prefrontal cortex. I. Single-neuron activity in orbitofrontal cortex compared with that in pyriform cortex. J Neurophysiol 74:733-750.

Schoenbaum G, Setlow B (2001) Integrating orbitofrontal cortex into pre- 
frontal theory: common processing themes across species and subdivision. Learn Mem 8:134-147.

Schoenbaum G, Chiba AA, Gallagher M (1998) Orbitofrontal cortex and basolateral amygdala encode expected outcomes during learning. Nat Neurosci 1:155-159.

Schoenbaum G, Chiba AA, Gallagher M (1999) Neural encoding in orbitofrontal cortex and basolateral amygdala during olfactory discrimination learning. J Neurosci 19:1876-1884.

Schoenbaum G, Chiba AA, Gallagher M (2000) Rapid changes in functional connectivity in orbitofrontal cortex and basolateral amygdala during learning and reversal. J Neurosci 20:5179-5189.

Schoenbaum G, Nugent S, Saddoris MP, Setlow B (2002) Orbitofrontal lesions in rats impair reversal but not acquisition of go, no-go odor discriminations. NeuroReport 13:885-890.

Schoenbaum G, Setlow B, Nugent SL, Saddoris MP, Gallagher M (2003) Lesions of orbitofrontal cortex and basolateral amygdala complex disrupt acquisition of odor-guided discriminations and reversals. Learn Mem 10:129-140.
Setlow B, Holland PC, Gallagher M (2002) Disconnection of the basolateral amygdala complex and nucleus accumbens impairs appetitive Pavlovian second-order conditioned responses. Behav Neurosci 116:267-275.

Setlow B, Schoenbaum G, Gallagher M (2003) Neural encoding in ventral striatum during olfactory discrimination learning. Neuron 38:625-636.

Thorpe SJ, Rolls ET, Maddison S (1983) The orbitofrontal cortex: neuronal activity in the behaving monkey. Exp Brain Res 49:93-115.

Tremblay L, Schultz W (1999) Relative reward preference in primate orbitofrontal cortex. Nature 398:704-708.

Tremblay L, Hollerman JR, Schultz W (1998) Modifications of reward expectation-related neuronal activity during learning in primate striatum. J Neurophysiol 80:964-977.

Williams GV, Rolls ET, Leonard CM, Stern C (1993) Neuronal responses in the ventral striatum of the behaving macaque. Behav Brain Res 55:243-252.

Yanagimoto K, Maeda H (2003) The nucleus accumbens unit activities related to the emotional significance of complex environmental stimuli in freely moving cats. Neurosci Res 46:183-189. 